\title{
Emerging roles of translational control in circadian timekeeping
}

\author{
Violeta Castelo-Szekely ${ }^{\mathrm{a}}$, David Gatfield ${ }^{\mathrm{a}, *}$ \\ ${ }^{a}$ Center for Integrative Genomics. University of Lausanne. Genopode, 1015, Lausanne, Switzerland.
}

\begin{abstract}
A large part of mammalian physiology and behaviour shows regular daily variations. This temporal organisation is driven by the activity of an endogenous circadian clock, whose molecular basis consists of diurnal waves in gene expression. Circadian transcription is the major driver of these rhythms, yet post-transcriptional mechanisms, some of which occur in response to systemic cues and in a tissue-specific fashion, have central roles in ultimately establishing the oscillatory gene expression programme as well. Regulatory control that occurs at the level of translation is emerging as an important player in the generation and modulation of protein accumulation rhythms. As a mechanism, translation lies at a privileged position to integrate genetically encoded rhythmic signals with other, external and internal stimuli, including nutrient-derived cues. In this review, we summarise our current knowledge of how diurnal control of translation affects both bulk protein levels and gene-specific protein biosynthesis. We discuss mechanisms of regulation, in particular with regard to the complex interplay between circadian cycles and feeding/fasting cycles, as well as emerging roles for upstream open reading frames (uORFs) in clock control.
\end{abstract}

Keywords: Circadian clock, Translation, Ribosome biogenesis, Feeding/fasting cycles, uORFs

\footnotetext{
* Corresponding author

Email address: David.Gatfield@unil.ch (David Gatfield)
} 


\section{Introduction}

Environmental changes can represent threats to be avoided and opportunities to be seized predicting them thus lies in an organism's best interest. This also holds true for the sharp, yet foreseeable 24-h periodic changes that are associated with the rotation of the Earth around its axis

5 and that engender daily rhythms in light and temperature conditions, humidity, food availability, predator exposure and other parameters. It is thus not surprising that most living beings have evolved sophisticated endogenous time-keeping devices, known as circadian clocks, to anticipate daily variations in living conditions and to synchronize their behavior, physiology and metabolism to geophysical time.

A spectrum of model organisms - ranging from cyanobacteria to plants, fungi, insects and mammals - has served scientists to illuminate from various angles, how nature has constructed its clocks and, given that circadian clocks appear to have arisen several times in evolution [1], how this occurred on independent occasions. While many of the genes and proteins that make up the core clock mechanism differ across evolutionarily distant organisms, it is noteworthy that most currently known circadian clocks rely on a similar molecular architecture for the generation of self-sustained oscillations in gene expression, which is through negative feedback loops in transcription [2]. In the simplest form, the typical core clock architecture thus consists of clock genes encoding transcriptional activators that drive the expression of clock genes encoding transcriptional repressors. Through physical interactions, the repressor proteins inhibit the activators to suppress further repressor synthesis, and due to their intrinsic instability, repressor mRNA and protein abundances rapidly drop below the threshold required for autorepression, clearing the way for a new cycle. Of note, it was the discovery of this mechanism, along with the identification of the first core clock genes in Drosophila, that earned the 2017 Nobel Prize in Physiology or Medicine.

With rhythmic transcription driving the core clock, the simplest way to relay timing information to downstream effector pathways would be by transcriptional mechanisms as well. A wealth of studies dedicated to the identification of mRNAs whose abundance periodically changes over the day, as well as the detailed understanding of the complex molecular events occurring at rhythmically active promoters [3], indeed leave little doubt that rhythmic transcription is key to generating the majority of cyclic gene expression in most organisms and cell types. However, there are notable exceptions. A radical revision of the transcription-centric model came from the findings that the cyanobacterial clocks can operate in the absence of any transcription at all and that self-sustained rhythmic phosphorylation of the essential clock component KaiC even occurs in vitro using recom- 
binant clock proteins and ATP [4]. Transcription-independent molecular rhythms have also been observed in human erythrocytes. In these naturally nucleus-free cells, antioxidant proteins known 35 as peroxiredoxins undergo changes in their redox status, which show self-sustained, entrainable, and temperature-compensated 24-hour periodicity in the absence of transcription and translation [5]. In the wake of such surprising discoveries (and as a consequence of the technical advances made in the fields of high-throughput nucleic acid sequencing and proteomics), the longstanding concept which places daily changes in transcriptional activity at the heart of rhythmic gene expression was revisited as well. A more nuanced picture has arisen that accords a significant role to post-transcriptional mechanisms in the generation of rhythmic clock outputs in mammals. Furthermore, it has become clear that a significant proportion of RNA and protein oscillations is not necessarily a direct output of the local, cellular clock, but rather driven by systemic cues, in particular by signals related to feeding.

This review aims at presenting and discussing our current knowledge of how one of the implicated post-transcriptional mechanism, i.e. translation, can generate, modulate, and sustain circadian rhythms. Our main focus will lie on the mammalian circadian system. Before we delve into the details, however, we shall take this opportunity for a brief digression into findings from two of the more exotic organisms that have been used in circadian clock research, namely green algae and marine dinoflagellates. It is in these systems that the first, and most compelling evidence for translationally driven clock activity was collected many years ago. An important case is provided by the green algae Acetabularia, which is an organism that is unicellular, yet the giant single cell consists of a rhizoid that contains the cell's single nucleus, a long stalk, and an umbrella of branches that can fuse into a cap. The rhizoid allows complete cellular regeneration when its cap is removed, and the caps of ${ }_{55}$ two Acetabularia cells can be experimentally exchanged, explaining why this organism has served as an important model species in early cell biological research. Interestingly, it was found that isolated caps would survive for several weeks without a nucleus, yet rhythmic photosynthesis would continue [6]. When an out-of-phase nucleus was reintroduced into such a plant, it was the rhythmic phase of the cytoplasmic photosynthetic activity that entrained the nuclear clock, rather than the inverse

${ }_{60}[7,8]$. Later work in this system established a distinct role of translation in rhythm generation [9]. A second, historically influential organism is Gonyaulax polyedra. This marine dinoflagellate produces circadian oscillations in nocturnal bioluminescence through the activity of three proteins that are regulated through rhythmic translation $[10,11]$ and that can be phase-shifted through translational inhibitors [12]. Subsequent work identified a specific protein, termed CCTR for Circadian-Controlled 
Translational Regulator, that shows daily cycles in binding activity to an RNA element located in the $3^{\prime}$ untranslated region of the circadianly expressed luciferin binding protein, thereby repressing its translation during the day [13]. The two examples, Acetabularia and Gonyaulax, thus show that in certain extreme cases nature appears to have placed regulated translation even at the center of the cellular core clock mechanism.

\section{Architecture of the mammalian circadian system}

The mammalian circadian system is hierarchically organised, with peripheral clocks in virtually every cell of the organism that are synchronised through a master pacemaker located in the brain's suprachiasmatic nuclei (SCN). The SCN receives photic signals from the environment via the retinohypothalamic tract connecting to the photosensitive retinal ganglion cells, thereby entraining internal timing to geophysical time (reviewed in [14]). The main molecular components of the mammalian cellular clockwork are transcriptional activators and repressors interlocked in negative feedback loops. In the main loop transcription factor heterdimers formed by CLOCK (or its brain paralogue NPAS2) and BMAL1 (also known as ARNTL) activate the transcription of target genes, including their own repressors, encoded by Period (Per1, Per2, Per3) and Cryptochrome (Cry1, Cry2) genes. PER:CRY complexes translocate to the nucleus and repress CLOCK:BMAL1 activity until their degradation allows a new activation cycle to start (Figure 1). In a secondary, interconnecting loop, CLOCK:BMAL1 drive the expression of Rev-erb (Rev-erb $\alpha$, Rev-erb $\beta$ ) and Ror (Ror $\alpha$, Ror $\gamma$ ) genes that, in turn, repress and activate Bmal1 transcription, respectively. Additionally, a multitude of post-translational modifications acting on the above clock components play important roles in various steps of the mechanism as well [15]. The rhythmic transcriptional activities that constitute the core clock mechanism are also responsible for the transcription of many other genes transcriptome-wide. These are termed clock output or clock-controlled genes (CCGs). Depending on cell type, CCGs can represent a sizeable proportion of the transcriptome. CCG repertoires are shaped in a highly tissuespecific fashion due to the co-regulation of genes by circadian and tissue-specific transcription factors, as well as the influence of systemic cues such as temperature and feeding that can differentially affect organs as well.

\section{Post-transcriptional mechanisms contribute to shaping gene expression rhythms}

Transcriptomics studies have shown that in organs such as brain, heart or kidney, around $4-12 \%$ of expressed genes show daily oscillations in mRNA abundance. In liver (arguably the best studied 


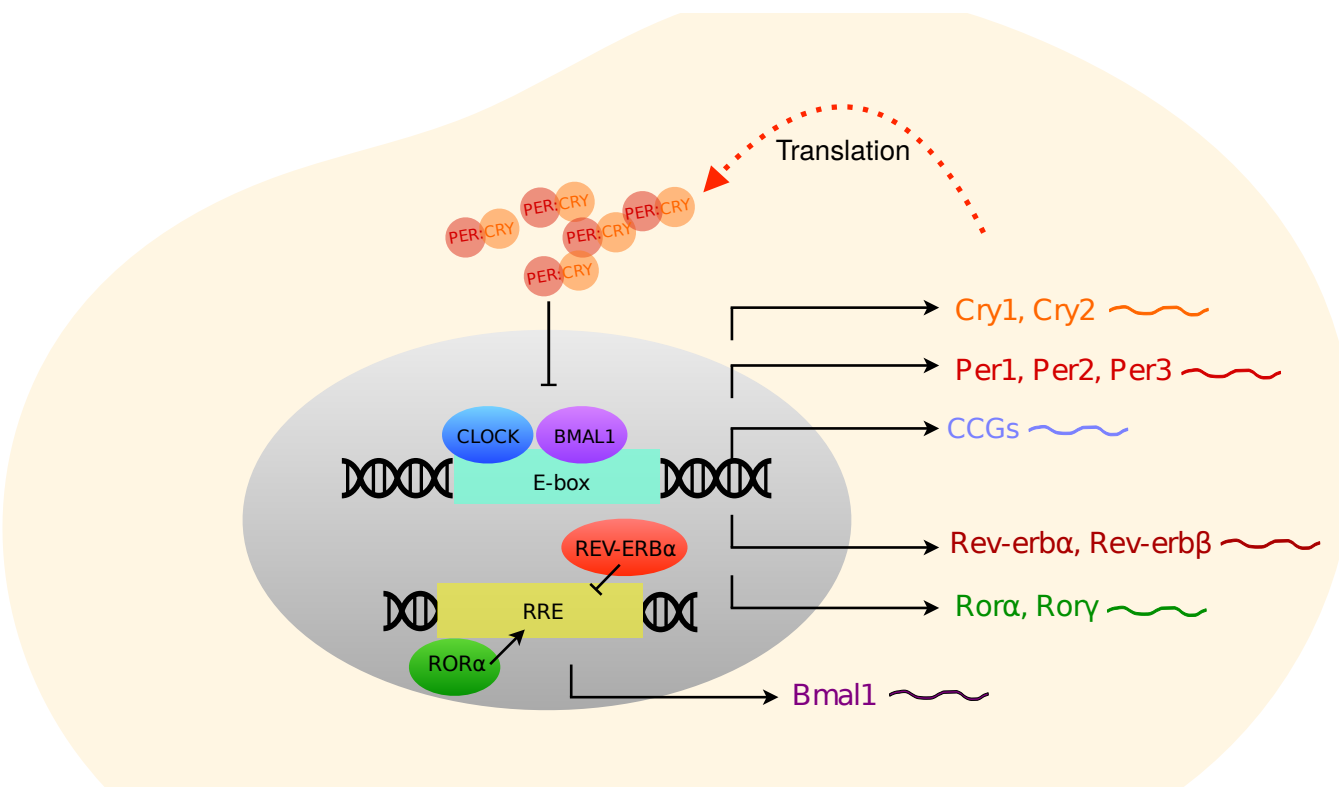

Figure 1: Simplified model of the transcriptional feedback loop of the mammalian circadian clock. This figure shows the main players of the interlocked negative transcriptional feedback loops that make up the mammalian clock. See text for details. peripheral model organ in the circadian field) the proportion is even as high as 20\% [16]. Most of these rhythms are thought to be driven transcriptionally. However, diurnal changes in mRNA stability appear to contribute quite significantly to establishing circadian transcriptomes as well. A few years ago, three complementary studies set out to determine which proportion of transcriptomal oscillations in mouse liver relied on post-transcriptional rather than on transcriptional mechanisms for their rhythmicity $[17,18,19]$. They led to the remarkable estimate that $\sim 25-70 \%$ of rhythmically accumulating mRNAs stemmed from genes that did not undergo oscillations in transcription. Of note, for reasons related to the definition of non-rhythmicity that have been discussed elsewhere $[20,21]$, there are grounds to believe that the extent of diurnally regulated transcript stability is more likely reflected by the more conservative of the above estimates.

Discrepancies have also become apparent in comparative analyses of mRNA and protein rhythms. Evidently, the proteome is furthest downstream from the initial transcription event, thus integrating the ensemble of regulation that impinges on mRNA stability, translation and protein stability. Early on, a pioneering small-scale proteomics study ( $<100$ proteins) had hinted at the notion that a considerable proportion of protein rhythms were not simply a propagation of oscillations occurring at the level of the encoding mRNAs [22]. More recently, deep proteomics analyses based on thousands 
of polypeptides in mouse liver have estimated that $\sim 5 \%$ of detectable proteins show diurnal variations, of which $20 \%$ [23] to $50 \%$ [24] are translated from constantly abundant, rather than rhythmic mRNAs. The rhythmicity that is observable at the proteomic level is expected to reflect significant contributions from protein synthesis, protein degradation and protein secretion, all of which could in principle have a time of day-dependent component. Diurnal patterns of post-translational protein modifications, such as phosphorylations [25, 26] and acetylations [27], play roles in the complex interplay between the clock and the proteome as well.

\section{Translational regulation of rhythmic gene expression}

\subsection{General principles of translational control}

Translation, i.e. the biosynthesis of proteins from mRNA templates, is a highly regulated process that is frequently considered to account for a large proportion of total cellular energy needs. Translation has the ability to integrate metabolic, stress-related and other cellular cues, allowing cells and organisms to respond quickly and reversibly to internal and environmental fluctuations and adapt their proteome accordingly [28]. Moreover, protein levels have been found to correlate better with the translational activity of mRNAs that with their abundances [29, 30, 31], highlighting the functional importance of translational control. In the following, we will first give an overview of the basic steps of eukaryotic translation and its regulation, before we discuss interactions with daily rhythms later in this review.

Four steps can be distinguished in translation: (1) ribosome scanning and initiation, (2) elongation, (3) termination and peptide release, and (4) ribosome dissociation and recycling. Translation initiation is considered to be the rate-limiting and most regulated step in the process (see [32] for an excellent review). Briefly, initiation requires the formation of the $43 \mathrm{~S}$ pre-initiation complex (PIC) at the start codon. The PIC is composed of a 40S small ribosomal subunit loaded with a methionine-charged initiator tRNA and $\mathrm{eIF} 2 * \mathrm{GTP}$, as well as a distinct set of additional initiation factors. In the classical, "cap-dependent" pathway, this protein complex is initially recruited to mRNAs by recognition of their $5^{\prime} \mathrm{m}^{7} \mathrm{G}$ cap through initiation factor eIF4E. eIF4E is a subunit of eIF4F, which also contains the scaffold protein eIF4G and eIF4A, a helicase that unwinds base-paired nucleotides within $5^{\prime}$ leader sequences. Of note, it is the recognition of the cap that is stringently controlled. To this end, eIF4E can be sequestered by eIF4E binding proteins (4E-BPs), making it unavailable for PIC recruitment and scanning and hence causing translational repression. The activity of 4E-BPs is regulated through phosphorylation; when hyperphosphorylated, they do 
not bind eIF4E and translation can ensue. A main player in 4E-BP phosphorylation is mTORC1, one of the two cellular complexes containing the mTOR kinase that is central to cell growth and proliferation and that responds to nutrient levels and mitogens. When mTORC1 is inactivated, the rapid dephosphorylation of 4E-BPs allows them to sequester eIF4E from eIF4F and inhibit capdependent translation. This model suggests a global decrease in (cap-dependent) translation that affects all cellular mRNA; however, we now know from several studies that the picture is much more fine-grained, as not all protein biosynthesis is affected equally [33, 34]. In particular, these studies have found that there are specific "mTOR-sensitive" mRNAs (for example the so-called 5' TOP mRNAs that will be discussed in detail later), but also additional non-TOP transcripts that can be grouped into different classes according to mRNA sequence motifs. The precise mechanisms of how these transcripts are regulated by mTOR remains poorly understood. Of note, an important general principle that is emerging from these and other studies is that many translation factors (in this case: eIF4E) that were previously considered to be part of a common, canonical translation machinery and required for most cellular translation, have turned out to be quite specific, or at least show strong dose dependence, for the translation of distinct mRNA subsets. The molecular basis and functional consequences of these differential requirements for initiation factors is still poorly understood, as is the role of the different translation factor paralogues that are found in mammals (e.g. two eIF4Gs, three eIF4Es, three 4E-BPs). Finally, while the above describes the situation for cap-dependent translation, it is emerging that many cellular mRNAs can recruit the translation apparatus cap-independently via internal ribosome entry sites (IRES) or through mechanisms that involve RNA modifications such as $\mathrm{N}^{6}$-methyladenosine $\left(\mathrm{m}^{6} \mathrm{~A}\right)$ (reviewed in [35]).

Once the 43S PIC is loaded on the mRNA, it scans for an appropriate initiation codon. In the canonical case this is an AUG whose initiation strength is determined by the local sequence context (in particular the well-known "Kozak sequence" [36]). Nevertheless, it is still challenging to precisely predict initiation sites only from mRNA sequence, because structures and elements in the $5^{\prime}$ leader sequence and the larger environment of the initiation codon influence start codon selection. One such class of sequences that will be discussed later in the context of circadan gene expression are the upstream open reading frames (uORFs), which are short, translated sequences within the $5^{\prime}$ UTR that compete with the main ORF for initiating ribosomes and thus generally have a repressive effect on CDS translation. An example for a 5'-borne RNA structure that will be discussed later, is the iron response element (IRE), which is found in mRNAs encoding proteins involved in iron, oxygen and energy metabolism. 
With regard to protein rhythmicity, it is conceivable that diurnal oscillations of translation efficiency could represent an important mechanism to establish circadian clock outputs, and this mechanism may explain (together with protein degradation that can also be regulated diurnally) how rhythmic protein accumulation is achieved in the absence of rhythmic mRNA abundance [37]. Translational regulation that is dependent on time of day could control protein levels globally, or it could act in a transcript-specific manner. As will be discussed in the following sections, the latter can be achieved through cis-elements that are present on a specific group of transcripts, while the former would affect the transcriptome as a whole by acting on general mechanisms of translational control. Translational regulation could also impact on rhythmic protein output by amplifying or reducing differences in the rhythmic transcriptomes across cell types and tissues, by adding robustness to the circadian system, or by introducing phase delays between mRNA and protein accumulation.

In the following sections, we will summarise our current knowledge of translational control of mammalian rhythmic expression, with a focus on which transcripts undergo diurnal translational control, on the underlying principles and mechanisms of regulation, and on the important interplay between the circadian and the feeding/fasting cycles in driving and sustaining these oscillations.

\subsection{Early and indirect evidence for time of day-dependent translational control}

In mouse tissues, translational regulation of rhythmic gene expression was initially estimated from the parallel measurement of protein and mRNA levels [22, 38]. Two-dimensional difference gel electrophoresis (2D-DIGE) from liver reported 60-135 rhythmic spots, corresponding to 10$20 \%$ of all detected ones. Of these, 39 protein were further identified by mass spectrometry (MS) and it was found that about half of them were encoded by arrhythmic mRNAs [22]. Two recent studies relying on advanced quantitative MS techniques (shotgun proteomics and in vivo SILAC, Stable Isotope Labeling by Amino acids in Cell culture) significantly improved detection and fidelity $[23,24]$, though it should be kept in mind that both still failed to reliably detect many lowly abundant proteins including the majority core clock components. Robles et al. [23] detected 3000 proteins, of which $6 \%$ showed robust daily oscillations with a broad distribution of phases. Parallel microarray-based analysis of the transcriptome revealed that one fifth of oscillating proteins did not show the corresponding oscillations at the transcript levels, indicative of widespread rhythmic post-transcriptional and post-translational regulation. Similarly, Mauvoisin et al. [24] identified around 5000 proteins, of which 195 (4\%) oscillated in abundance and, of those, about half did not show diurnal regulation of their corresponding mRNAs. Beyond liver, the circadian proteome has recently been interrogated in mouse SCN, where a little less than $2.5 \%$ of the 2000 detected 
proteins showed robust 24-hour oscillations, most of them without evidence for rhythmic mRNA [39]. In all three studies, the circadian proteomic landscape was enriched for specific biological processes, such as xenobiotic detoxification [23] and protein secretion [24] in liver, and mitochondrial oxidative phosphorylation in the SCN [39], indicating tissue specificity of post-transcriptional control. Moreover, the studies reported a phase delay from transcript to protein accumulation that was in the range of 5 to more than 8 hours $[23,24,39]$. In order for such relatively large differences in mRNA and protein peak times to occur, it is plausible that the mechanisms that regulate protein clearance - i.e. degradation and secretion - are also subject to diurnal control.

Circadian clock-control of protein expression that is independent from mRNA levels has also been inferred through the study of poly $(\mathrm{A})$ tail dynamics as a proxy of message translatability. In mouse liver, >200 transcripts exhibited 24-hour oscillations of poly(A) tail length [40], and although the majority was likely a consequence of rhythmic transcription, the study identified 42 transcripts ( $18 \%$ of all poly(A)-rhythmic mRNAs) whose daily changes in poly(A) tail length were not explained transcriptionally nor by steady-state mRNA level oscillations. It was thus concluded that they were under the control of diurnal cytoplasmic polyadenylation. Consistently, maximal tail length was correlated with the phase of highest expression of polyadenylation element binding proteins (CPEBs), as well as with peak accumulation of the encoded proteins [40].

While the ensemble of these studies is highly suggestive of widespread translational contributions to the establishment of protein rhythms, they did not interrogate the role of translation and daily changes in translational efficiency directly. It has been known since the 1960s that many ribosomes progress along one mRNA molecule simultaneously ('polysomes') [41] and until 10 years ago, the analysis of translation efficiencies still relied on traditional methodologies based on the ultracentrifugation of polysome-containing extracts on sucrose gradients, which separates mRNAs according to how many ribosomes they carry, followed by rather cumbersome fractionation and analysis. It is only with the development of ribosome profiling in 2009 that the analysis of translation rates has become truly high-throughput compatible [42]. In this technique, the polysome-containing extracts are directly treated with nuclease under conditions that degrade the majority of cellular RNAs but keep individual ribosomes ('monosomes') and a short fragment of protected mRNA, known as the footprint, intact. The footprints, whose $\sim 30 \mathrm{nt}$ sequences are diagnostic of transcript identity and position of the translated codon, are then purified, converted into sequenceable libraries and analysed by high-throughput sequencing. 


\subsection{Translational regulation of ribosome biogenesis}

In a study that still relied on the traditional methodology - microarray quantification of transcripts isolated from gradient-fractionated polysomes - Jouffe et al. analysed ribosome association

\subsection{Other pathways and transcripts undergoing diurnal translation regulation}

Transcripts encoding components of the translation machinery were by far the largest and most striking group of translationally rhythmic mRNAs in liver, but the ribosome profiling data uncovered 
two other distinct classes as well. These were, first, Translation Initiation of Short $5^{\prime}$ UTR Motifs (TISU) transcripts [45] and, second, iron-responsive element (IRE)-containing mRNAs [44] (Figure 2).

TISU mRNAs are characterised by an AUG initiation codon that is preceded by an extremely short $5^{\prime}$ UTR. This configuration at the mRNA $5^{\prime}$ end directs efficient, cap-dependent translation initiation and confers several characteristics, notably its relative independence of two factors implicated in most other, cap-dependent initiation events, eIF4A (a helicase resolving secondary structures) and eIF1 (a key factor in determining translation initiation fidelity). It has thus been hypothesised that TISU permits efficient cap-dependent translation initiation without scanning of the $5^{\prime}$ UTR for the start codon, a mechanism that would be advantageous when intracellular levels of eIF1 and eIF4A fluctuate [49]. Atger et al. provide evidence that this class of transcripts is rhythmically regulated at the protein synthesis level in mouse liver, reaching maximal translation efficiency at ZT 10 - an effect that appears to be dependent on feeding rhythms [45].

IREs are RNA stem-loop structures found in transcripts encoding for proteins involved in iron, oxygen and energy metabolism, and they constitute a well-known example of translational regulation [50]. IRE-containing Fth1, Ftl1 and Alas2 mRNAs are constant in abundance, but they show rhythmic translation with a peak and trough at around ZT 4 and ZT 12, respectively, that leads to protein oscillations, suggesting that iron metabolism is under diurnal control [44]. In all three messages, the IRE is located in the $5^{\prime}$ UTR, which confers regulation by the IRE/IRP system at the level of translation [50]. Briefly, in conditions of cellular iron deficiency (and also regulated through oxygen levels and other cues), iron-regulatory proteins IRP1 (also known as ACO1) and IRP2 (or IREB2) bind $5^{\prime}$ UTR IREs and repress translation initiation of iron storage (Fth1, Ftl1), heme biosynthesis (Alas2) and iron export (Slc40a1/Ferroportin) transcripts. IRP binding is precluded when iron levels are high, and the mRNAs can subsequently be translated. This autoregulatory mechanism plays a critical role in iron homeostasis. Mechanistically, it involves an Fe-S cluster that is assembled on IRP1, which converts it to cytosolic Aconitase that cannot anymore interact with the IRE; IRP2 is degraded by the proteasome in an oxygen- and iron-dependent fashion by a mechanism involving the protein FBXL5 [50]. Of note, at least one transcript, encoding transferrin receptor 1 (Tfrc), contains IREs in the $3^{\prime}$ UTR, which regulates the stability rather than the translation of the message [51]. Interestingly, while the transcription of the Tfrc gene is not rhythmic, its mRNA is, which suggests that the Tfrc transcript half-life undergoes daily changes [18] and that the mechanism underlying rhythmic IRE activity is not confined to translational control. How the 


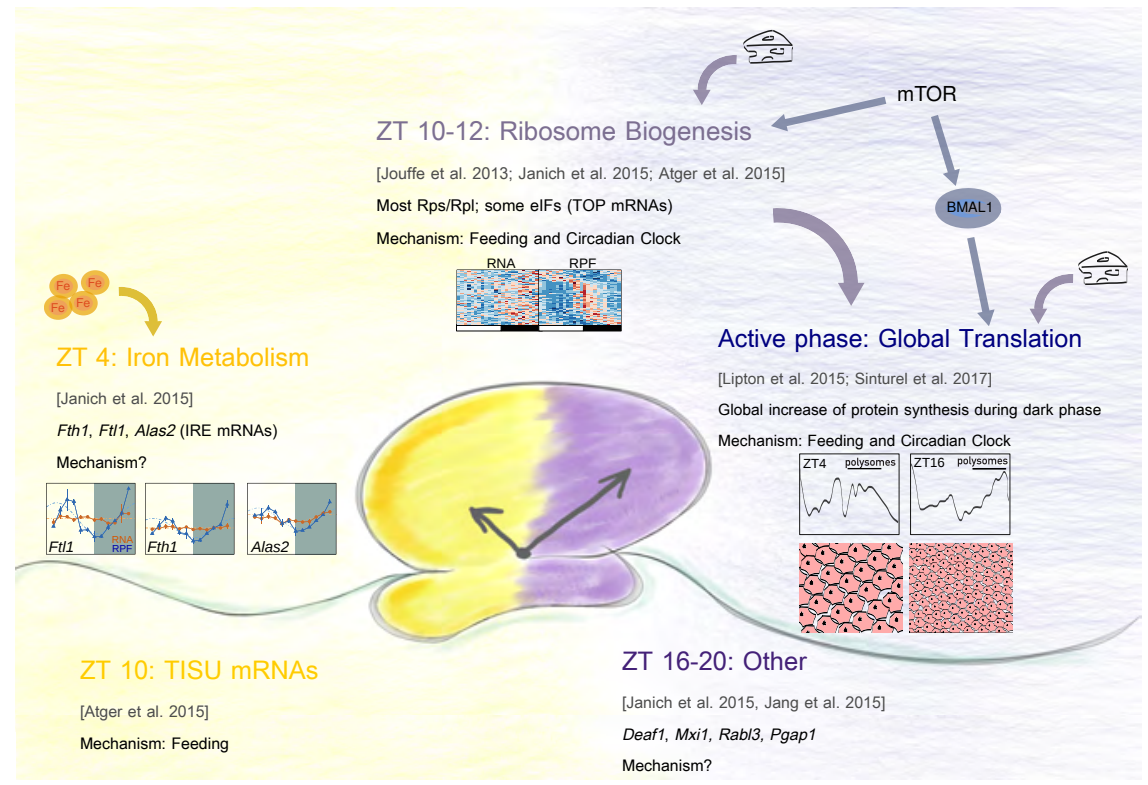

Figure 2: Multiple interactions between circadian gene expression and translation. Summary and overview of the main findings, pathways and mechanisms (if known) regarding the contribution of translation to diurnal protein expression. Rhythmic translation affects individual transcripts (e.g. mRNAs encoding proteins involved in iron metabolism; ribosomal protein mRNAs), but also seems to be a systemic property of liver gene expression that is intrinsically connected to feeding rhythms.

circadian clock might be involved in rhythmic regulation at the IRE awaits further investigation, in particular with respect to the involvement of IRPs (which are themselves constitutively expressed [44]), the precise requirement for a local vs. central clock to drive the oscillations, or the contribution of other, systemic cues (e.g. iron levels, food intake).

In summary, circadian translatome studies have shown that time of day-dependent control of protein synthesis acts on mRNAs that encode proteins with similar functions and that contain specific $5^{\prime}$ UTR motifs. These confer coordinated regulation, for example in response to systemic cues. Moreover, peaks in translational activity appear to have a preference for two predominant circadian phases, dawn and dusk. While the above data describes the situation in mammalian organs (mainly liver), it should be pointed out that similar features have been observed in studies of the diurnal translatomes of Drosophila neural clocks [52], of Arabidopsis thaliana [53], and in a human cell line, U2OS cells [54]. 


\section{Interplay between circadian clocks and feeding/fasting cycles - transcript-specific and global effects on translation}

Rhythms in gene expression in peripheral organs, both at the transcriptional and at the posttranscriptional level, not only reflect the activity of the local circadian clock. They also incorporate the inputs of other daily rhythmic cues, notably body temperature, feeding/fasting, hormonal signals, or sleep. The individual contribution of a particular physiological cue can be hard to discern, as they coalesce in the organism and feed back to each other. For example, body temperature is systemically controlled by the hypothalamus, including through circadian signals from the SCN, and temperature can entrain cell-autonomous peripheral oscillators through temperature-sensitive gene expression programs $[55,56,57,58,59]$. Similarly, feeding and fasting are intrinsically coordinated with wake/resting cycles by means of the endogenous clock [60], and in turn, the nutrient status can orchestrate circadian expression and function (reviewed in [61]; see also [62, 63] for recent studies). Historically, the liver has been the favourite model to study such peripheral organ properties - a bias that can be explained by this organ's large size, its easy dissectability, relatively homogeneous cell composition, and obviously its widespread and high amplitude circadian oscillations, most of which are related to metabolic functions. The temporal adjustment of metabolic (e.g. anabolic vs. catabolic pathways) and detoxification processes by the liver is necessary for the organism to ensure efficient use of available nutrients and disposal of xenobiotics. Therefore, liver is particularly suitable to study the relative contributions of feeding rhythms and circadian signals in coordinating diurnal gene expression. Briefly, an array of studies involving time-restricted feeding in full-body or liverspecific circadian clock-deficient mouse strains, has allowed the conclusion that feeding rhythms are the dominant driver of a significant proportion of hepatic gene expression rhythms. Early studies already showed that in mouse models lacking specifically the liver clock, approximately $90 \%$ of mRNA abundance rhythms were lost, yet the remaining 10\% (which included the core clock gene Per2) continued to cycle due to systemic cues [64,65]. Moreover, feeding-fasting cycles were recognised as the underlying driver of a large part of rhythmic gene expression in wild-type mice, and time-restricted feeding restored a significant fraction of hepatic mRNA oscillations in Cry1/Cry2 double-knockout mice that are deficient for a functional circadian clock [66]. Two recent studies have reported on the inverse experiment, i.e. the application of an arrhythmic feeding protocol in wild-type animals with an intact clock [67,68]. Greenwell et al. [68] observed that about $70 \%$ of mRNA rhythms were lost under these conditions, further underlining the exceptional importance of feeding-fasting cycles for hepatic gene expression rhythms. 
The translational upsurge of transcripts encoding ribosomal proteins and other components of the translation machinery that can be observed in the livers of night- or ad libitum-fed animals $[43,44,45]$ (see above) occurs at the light-to-dark transition, which also corresponds to the end of resting/fasting and the beginning of activity/feeding phases. The feeding/fasting cycle is indeed crucial for this regulation. As mentioned above, the affected mRNAs contain $5^{\prime}$ TOP motifs that mediate regulation by the nutrient-sensitive mTORC1 pathway [46]. Of note, $5^{\prime}$ TOP mRNAs retain translational oscillations in the livers of clock-deficient mice subjected to night-restricted feeding [45], uncovering feeding (or fasting) as the main driver of $5^{\prime}$ TOP rhythms in liver. It is noteworthy that in mice fed ad libitum RP mRNA translation peaked already at ZT 10, i.e. 1-2 hours before animals typically begin food intake under theses conditions [69]. One may thus speculate that the translational upregulation is more than a simple acute response to food intake. The complexity in the interactions between feeding and circadian signals thus likely also extends to the translational level. For example, TORC1 expression itself is clock-controlled in liver [43], conceivably resulting in circadian gating of the feeding response.

The above examples describe daily changes in translation efficiency that affect small groups of transcripts and that are relative to the bulk of the transcriptome whose translation rate is assumed to be constant across time points. It is important to note that it cannot be easily determined from ribosome profiling data whether this assumption of constant global translational activity is true. The reason is that in these datasets, global changes in translation rate are indistinguishable from differences in sequencing depth; they are normalised out by the conventional analytical methods. Nevertheless, there are many hints, including from ribosome profiling studies, that point towards such global effects. First, it is known that under conditions where mTOR regulates $5^{\prime}$ TOP transcripts, global translation responds as well, an effect that is mediated through control of the eIF4G-eIF4E interaction [47]. Second, diurnal control of translation at a global scale may be expected as a consequence of the increased translation of ribosomal components that is indicative of ribosome biogenesis during the dark phase [43, 44, 45] (Figure 2).

Indeed, there are reports of daily changes in liver polysome profiles that go back to the 1960's, showing that in rats the polysome-to-total ribosome ratio increases by $\sim 25 \%$ during the night [70], compatible with recent observations from mouse liver [43, 71]. Consistent with translation rates that globally fluctuate along the day, Sinturel et al. recently reported that the size of the individual hepatocyte cells and the mass and macromolecular content of the whole mouse liver undergo strong daily oscillations in a feeding-dependent manner [71]. These observations are in line with earlier evidence 
of size fluctuations in subcellular structures [72], and with reports of daily liver mass changes in birds [73]. Sinturel et al. further showed that hepatic protein content rhythms were accompanied with ribosome assembly cycles that were driven precisely through the rhythmic production of ribosomal proteins, which then associate with rRNA that is synthesised continuously and in excess. Organ size oscillations appear to be specific to liver and were not observed in other, less metabolically active organs such as kidney [71], consistent with the lack of RP translation rhythms in this tissue [48]. Taken together, these studies show that in liver ribosome availability is rate limiting for protein synthesis, and that translational capacity undergoes global, daily changes. The activities of the circadian clock and feeding cycles allow for an alignment of high demand for protein synthesis to the time of nutrient abundance. We know that the misalignment between feeding/fasting and circadian cycles - that can occur in humans due to "social jet lag" or shift work - is associated with inadequate energy expenditure, increased risk for metabolic diseases [74], weight gain [75, 76], and mortality in rodents [77], explaining the considerable current interest in the benefits of time-restricted feeding diets $[74,78,79]$. It is quite plausible that the above mechanisms acting at the translational level play a critical role in this complex network of interactions.

\section{Growing complexity of interactions between the clock and the mTOR pathway}

In addition to the examples and experiments described above, a sizeable number of studies has demonstrated that the mTOR pathway and the core clock mechanism interact at many other levels as well. These interactions occur bidirectionally, i.e. from mTOR to the clock, and from the clock to mTOR. A specific role lies in the SCN, where mTOR signaling is involved in photic entrainment of the central oscillator. This pathway has been molecularly quite well characterised, and we shall describe it in the next section. Here, we will first present other examples and intriguing observations that all point to important functions of mTOR in rhythmic regulation and vice versa. Nevertheless, it is probably fair to say that we still understand too little to be able to place all findings in a single coherent model.

Lipton et al. [80] uncovered an intriguing specific mechanism how the clock machinery could directly feed into the translation apparatus via mTOR. The study suggests that the core clock transcription factor BMAL1 leads a second life in the cytosol, where it physically associates with the translation machinery and with the $5^{\prime}$ cap structure of mRNAs to increase protein biosynthesis. The mechanism involves rhythmic phosphorylation of BMAL1 through one of the main mTOR effector kinases, ribosomal S6 protein kinase 1 (S6K1). Metabolic labelling and co-immunoprecipitation 
experiments suggest that BMAL1 is most potent at activating translation towards the end of the active (night) phase. It is intriguing that this mechanism appears to be not only active in the animal (notably in liver). It is also detectable in cultured cells, despite the absence of rhythmic feeding cues that could entrain mTOR in this system, thus indicating a genuine cellular circadian activity. It will be exciting to study whether this mechanism truly affects global translation, or whether it has a preference for specific transcripts.

The recent study by Wu et al. places a specific clock protein, PER2, upstream of/within the mTOR mechanism [81]. PER2 is thus identified as a scaffold protein that tethers mTOR together with partner proteins Tsc1 and Raptor into a complex, in which mTOR is in a repressed state. Because Per2 expression is downstream of glucagon signaling that is active during fasting, the authors propose that PER2 acts as a connection between fasting and mTOR that impacts protein synthesis, autophagy and cell proliferation.

Finally, mTOR signaling feeds into the clock not only in the SCN (next section), but in a number of clock model systems and cell types. A comprehensive study by Ramanathan et al. used a variety of pharmacological and genetic mTOR gain- and loss-of-function models, as well as different cell and tissue types, to demonstrate that activation of mTOR shortens period and augments amplitude [82]. Through which specific clock protein(s) and mechanisms these effects are mediated is, however, still an outstanding question. Initially identified candidates are CRY1, CLOCK and BMAL1, whose abundances all increase when mTOR is constitutively activated [82, 83]. Recent findings that place mTORC1-mediated translational regulation of Period mRNAs downstream of feeding-mediated insulin signaling make the mechanism even more complex [84].

\section{Specific roles of the mTOR pathway in the SCN}

Light is the main cue regulating the SCN clock. It is perceived by the retina and projected through the retinohypothalamic tract to neurons on the ventral side of the SCN, where it activates intracellular signaling pathways that control clock gene expression and lead to clock resetting. mTOR is one of the pathways implicated in the mechanism. Light exposure during the subjective night (but not during the subjective day) thus triggers the dephosphorylation of mTOR effectors, including 4E-BP1 [85], and the in vivo infusion of the mTOR inhibitor rapamycin modifies the phase-shifting effects of light on behavioural rhythms and attenuates the light-induced expression of PER1 and PER2 [86]. Classical mTOR targets (i.e. 5' TOP mRNAs) also showed light-induced expression, thus indicating that the mTOR pathway functions to mediate light-evoked protein trans- 
lation and clock entrainment in the SCN. Cao and colleagues further dissected the specific role of rhythmic mTOR activity in the SCN, starting with the observation that 4E-BP1 shows rhythmic phosphorylation even in the absence of light [87]. Rhythmic 4E-BP1 activity leads to translational activation/repression cycles on the mRNA encoding for vasocative intestinal peptide (VIP), which has an important signaling role between ventral and dorsal SCN cells. Indeed, animals deficient for 4E-BP1 exhibit higher VIP levels and circadian behavioural phenotypes, as they re-entrain faster to a shifted light/dark cycle and are more resistant to the rhythm-disruptive effects of constant light. Moreover, the genetic inactivation of mTOR specifically in Vip-expressing neurons resulted in weakened synchronization between SCN cells and weakened circadian behavioural rhythmicity under various photic conditions (e.g. in constant light), further corroborating the specific and important role of mTOR signaling in this population of master clock neurons [88].

\section{Core clock regulation through upstream open reading frames (uORFs)}

Beyond the above examples where rhythmic protein biosynthesis is engendered through time of day-dependent changes in translation rates, translational regulation is emerging as relevant for the circadian clock also via other mechanisms. In this respect, the codon resolution that ribosome profiling data provides with regard to the translated portion of the transcriptome, has been instrumental in uncovering the activity of a class of regulatory $5^{\prime}$ UTR elements known as upstream open reading frames (uORFs). uORFs are short translated sequences that are either fully embedded in the $5^{\prime}$ UTR of mRNAs or partly overlap the main protein coding sequence (CDS) [89]. They initiate from canonical AUG start codons, but frequently also from non-canonical near-AUG codons [90]. It has been estimated that $\sim 50 \%$ of mammalian transcripts contain at least one uORF [89] and many transcripts contain several, attesting to the high regulatory potential of this class of elements. uORFs can act on CDS protein production via various mechanisms. In the vast majority of cases, this does not appear to implicate the uORF micropeptide that is produced, but rather a regulatory role of uORF translation [91]. As described earlier in the review, most eukaryotic translation involves scanning the mRNA from the $5^{\prime}$ cap in $3^{\prime}$ direction until it identifies a suitable initiation codon. In general, the presence of a uORF will thus hinder ribosomes from scanning through to the CDS. Ribosomes may thus restart translation, or skip the uORF altogether, which are two inefficient processes known as translation reinitiation and leaky scanning, respectively [91]. Consequently, the translation of uORFs is generally associated with lower protein synthesis [89, 91, 92].

There is precedence that uORFs plays a role in the clock from Neurospora crassa, where uORF 

FRQ [93], and the evidence for functional roles in the mammalian clock is accumulating, too. In liver, several core clock transcripts (Bmal1, Clock, Cry1, Rev-erb $\alpha$, Rev-erb $\beta$ ) displayed extensive ribosome footprint coverage in the $5^{\prime}$ UTR indicative of active uORF translation, and in some cases (a prominent example is Rev-erb $\alpha$ ) the translation rate of the uORF was even higher than that of the CDS [44]. The liver data [44] and complementary observations in U2OS cells [54] both suggest that the translation of these uORFs occurs constitutively and does not introduce additional rhythmic regulation on the oscillating mRNA. Conceivably, however, the constant blunting of clock protein biosynthesis across the cycle would impact the kinetics of clock protein accumulation and the stoichiometry between the different clock components, for example of negative vs. positive regulators. Moreover, one may speculate that differential uORF usage across cell and tissue types, or in response to physiological cues, could provide a means of modulating clock parameters without changes in clock gene transcription. A systematic investigation of the functions of each individual uORF is still missing. Nonetheless, for one case, in the gene Nr1d1/Rev-erb $\alpha$, uORF deletion increased protein expression and the magnitude of NR1D1 reporter oscillations, though the uORF was not critical for NR1D1 rhythmicity per se [44]. Moreover, the loss-of-function of DENR, a protein involved in translation reinitiation after uORF usage [94], led to the robust shortening of circadian period by 1-1.5 hours in NIH3t3 fibroblasts [44]. While it is unclear, which specific reinitiation event(s) underlie(s) the phenotype, the recent genome-wide identification of DENRsensitive transcripts has identified Clock itself as one of the candidates [95]. The study uncovered a complicated translational landscape within the Clock $5^{\prime}$ UTR. Two highly translated uORFs thus reduce CLOCK protein production almost by factor 2 . The downstream uORF overlaps by several codons (but in a different frame) with the annotated CLOCK CDS, and it is capable of triggering reinitiation from a downstream AUG start codon, giving rise to a CLOCK variant that is 9 amino acids shorter than the annotated CLOCK sequence predicts. In the absence of DENR, CLOCK protein output is markedly reduced by $\sim 30 \%$. While the in vivo implications of these regulatory events have not yet been studied, one may speculate that they allow for a regulated adjustment of protein synthesis under particular physiological conditions. One hypothesis is based on the original finding that DENR expression increases in cells at high density (hence its original name, Density Regulated Protein [96]). One may thus hypothesise that some of the reported effects of cell density on circadian rhythmicity $[97,98]$ operate through this mechanism (Figure 3 ). 


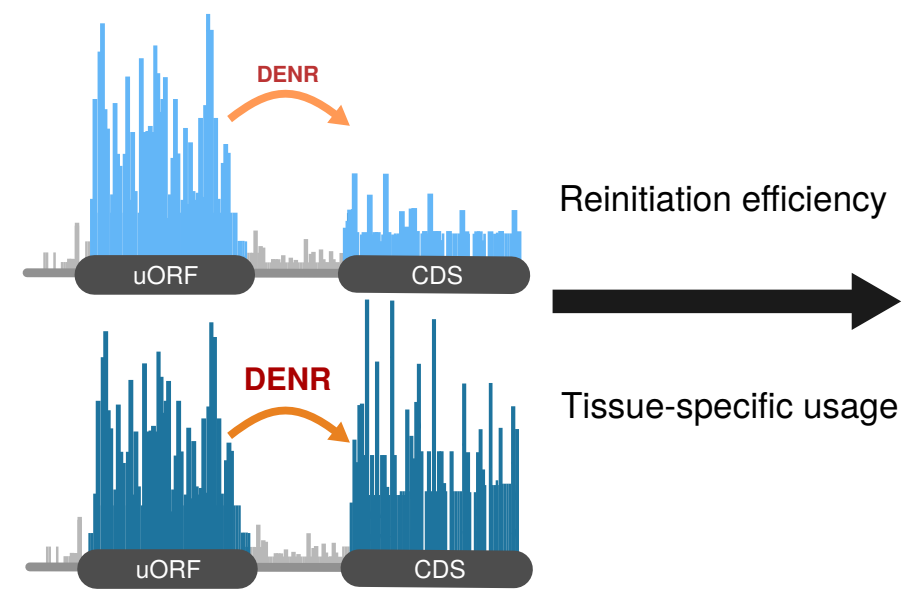

\section{Core-clock stoichiometry?}

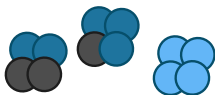

Clock properties?

(period, phase, ...)

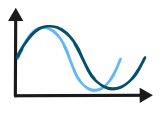

Links to cell density?

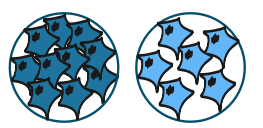

Figure 3: Regulation of core clock protein biosynthesis and circadian function through uORFs. Ribosome profiling allows the annotation of translated $\mathrm{UORFs}$ and determining how they affect the regulation of CDS translation efficiency. Several core clock transcripts contain one or several uORFs. Changes in reinitiation efficiency (for example due to the levels of reinitiation factors such as DENR) and/or tissue-specific uORF usage could regulate the levels of core clock proteins and thus complex stoichiometry, altering clock parameters in a way that is dependent on organ, physiological condition, or other cues. Moreover, since DENR expression increases in cells at high density, uORF usage and reinitiation efficiency could represent a link between circadian rhythmicity and cell density.

\section{Final considerations}

For a long time, translation was considered a necessary, yet almost default and largely uninteresting step on the way from gene to protein. Through ribosome profiling we now have the possibility to query specifically, directly and quantitatively the translational landscape of cells and organs. Our understanding of the various contributions that regulated translation makes to rhythmic gene expression has since exploded, yet we have clearly only begun to understand some of the interactions between clocks and the translation apparatus. As a mechanism, translation lies at a privileged position right where the protein is made, the main actor of most cellular functions. Translation would thus be optimally suited as a step where the gene expression programs that are 'hard-wired' through transcription can be rapidly integrated with other, acute information. The finding that a substantial fraction of rhythmic translation events is connected to time-of-feeding validates this idea.

Regulated translation impinges in other ways on the clock. One such example are uORFs, which were for a long time considered a rather exotic gene regulatory phenomenon of not more than anecdotal importance. We now know that thousands of transcripts, including many rhythmically expressed ones, contain regulatory uORFs. Nevertheless, their functions and the mechanisms by 
which they act are only starting to become visible. In the future, this understudied class of regulatory elements may therefore lead to the discovery of some surprising biology.

Finally, there are other interactions between translation and clocks that are fascinating yet still poorly explored in molecular terms. One example that this review did not cover, is the finding that it matters to circadian clock proteins from which codons they are translated. This phenomenon has been described in cyanobacteria [99], Neurospora crassa [100] and more recently also in flies [101]). In all cases, changing the codons within the CDS of clock components to different, synonymous codons that would be predicted to improve translation, led to defects in clock function. It would thus seem that on top of the conventional 3-letter codon table that specifies amino acids, there is an additional, more complex code interpreted by the ribosome. Very likely, this code plays a role in the coordination of the speed of protein biosynthesis with the folding of the nascent polypeptides [102].

\section{Acknowledgements}

Research in the authors' lab is funded by the Swiss National Science Foundation through the National Centre of Competence in Research (NCCR) RNA \& Disease (grant no. 141735) and individual grant 179190.

\section{References}

\section{References}

[1] M. Rosbash, The implications of multiple circadian clock origins, PLOS Biology 7 (2009) 1-5. doi:10.1371/journal.pbio.1000062.

[2] S. Brown, E. Kowalska, R. Dallmann, (re)inventing the circadian feedback loop, Developmental Cell 22 (2012) 477 - 487. doi:10.1016/j.devcel.2012.02.007.

[3] J. S. Takahashi, Transcriptional architecture of the mammalian circadian clock, Nature Reviews Genetics 18 (2017) 164-179. doi:10.1038/nrg.2016.150.

[4] M. Nakajima, K. Imai, H. Ito, T. Nishiwaki, Y. Murayama, H. Iwasaki, T. Oyama, T. Kondo, Reconstitution of circadian oscillation of cyanobacterial KaiC phosphorylation in vitro, Science 308 (2005) 414-415. doi:10.1126/science.1108451. 
[5] J. S. O'Neill, A. B. Reddy, Circadian clocks in human red blood cells, Nature 469 (2011) 498-503. doi:10.1038/nature09702.

[6] B. M. Sweeney, F. T. Haxo, Persistence of a photosynthetic rhythm in enucleated acetabularia, Science 134 (1961) 1361-1363. doi:10.1126/science.134.3487.1361.

[7] J. C. Woolum, A re-examination of the role of the nucleus in generating the circadian rhythm in acetabularia, Journal of Biological Rhythms 6 (1991) 129-136. PMID: 1773086, doi:10.1177/074873049100600203.

[8] E. Schweiger, H. G. Wallraff, H. G. Schweiger, Endogenous circadian rhythm in cytoplasm of acetabularia: Influence of the nucleus, Science 146 (1964) 658-659. doi:10.1126/science.146.3644.658.

[9] R. Hartwig, M. Schweiger, R. Schweiger, H. G. Schweiger, Identification of a high molecular weight polypeptide that may be part of the circadian clockwork in Acetabularia, Proc. Natl. Acad. Sci. U.S.A. 82 (1985) 6899-6902.

[10] D. Morse, P. M. Milos, E. Roux, J. W. Hastings, Circadian regulation of bioluminescence in Gonyaulax involves translational control, Proc. Natl. Acad. Sci. U.S.A. 86 (1989) 172-176.

[11] C. H. JOHnSON, J. F. ROEBER, J. W. HASTINGS, Circadian changes in enzyme concentration account for rhythm of enzyme activity in gonyaulax, Science 223 (1984) 1428-1430. doi:10.1126/science.223.4643.1428.

[12] W. R. Taylor, J. C. Dunlap, J. W. Hastings, Inhibitors of protein synthesis on 80s ribosomes phase shift the Gonyaulax clock, J. Exp. Biol. 97 (1982) 121-136.

[13] M. Mittag, D. H. Lee, J. W. Hastings, Circadian expression of the luciferin-binding protein correlates with the binding of a protein to the 3' untranslated region of its mrna, Proceedings of the National Academy of Sciences 91 (1994) 5257-5261. doi:10.1073/pnas.91.12.5257.

[14] J. A. Mohawk, C. B. Green, J. S. Takahashi, Central and peripheral circadian clocks in mammals, Annu Rev Neurosci 35 (2012) 445-462. doi:10.1146/annurev-neuro-060909-153128.

[15] A. Hirano, Y.-H. Fu, L. J. Ptacek, The intricate dance of post-translational modifications in the rhythm of life, Nature Structural \& Molecular Biology 23 (2016) 1053-1060. doi:10.1038/nsmb.3326. 
[16] R. Zhang, N. F. Lahens, H. I. Ballance, M. E. Hughes, J. B. Hogenesch, A circadian gene expression atlas in mammals: Implications for biology and medicine, PNAS 111 (2014) 1621916224. doi:10.1073/pnas.1408886111.

[17] N. Koike, S.-H. Yoo, H.-C. Huang, V. Kumar, C. Lee, T.-K. Kim, J. S. Takahashi, Transcriptional Architecture and Chromatin Landscape of the Core Circadian Clock in Mammals, Science 338 (2012) 349-354. doi:10.1126/science.1226339.

[18] G. Le Martelot, D. Canella, L. Symul, E. Migliavacca, F. Gilardi, R. Liechti, O. Martin, K. Harshman, M. Delorenzi, B. Desvergne, W. Herr, B. Deplancke, U. Schibler, J. Rougemont, N. Guex, N. Hernandez, F. Naef, Genome-Wide RNA Polymerase II Profiles and RNA Accumulation Reveal Kinetics of Transcription and Associated Epigenetic Changes During Diurnal Cycles, PLoS Biol 10 (2012). doi:10.1371/journal.pbio.1001442.

[19] J. S. Menet, J. Rodriguez, K. C. Abruzzi, M. Rosbash, Nascent-Seq reveals novel features of mouse circadian transcriptional regulation, eLife 1 (2012). doi:10.7554/eLife.00011.

[20] S. Lück, P. O. Westermark, Circadian mrna expression: insights from modeling and transcriptomics, Cellular and Molecular Life Sciences 73 (2016) 497-521. doi:10.1007/s00018-015-20722 .

[21] M. E. Hughes, K. C. Abruzzi, R. Allada, R. Anafi, A. B. Arpat, G. Asher, P. Baldi, C. de Bekker, D. Bell-Pedersen, J. Blau, S. Brown, M. F. Ceriani, Z. Chen, J. C. Chiu, J. Cox, A. M. Crowell, J. P. DeBruyne, D.-J. Dijk, L. DiTacchio, F. J. Doyle, G. E. Duffield, J. C. Dunlap, K. Eckel-Mahan, K. A. Esser, G. A. FitzGerald, D. B. Forger, L. J. Francey, Y.-H. Fu, F. Gachon, D. Gatfield, P. de Goede, S. S. Golden, C. Green, J. Harer, S. Harmer, J. Haspel, M. H. Hastings, H. Herzel, E. D. Herzog, C. Hoffmann, C. Hong, J. J. Hughey, J. M. Hurley, H. O. de la Iglesia, C. Johnson, S. A. Kay, N. Koike, K. Kornacker, A. Kramer, K. Lamia, T. Leise, S. A. Lewis, J. Li, X. Li, A. C. Liu, J. J. Loros, T. A. Martino, J. S. Menet, M. Merrow, A. J. Millar, T. Mockler, F. Naef, E. Nagoshi, M. N. Nitabach, M. Olmedo, D. A. Nusinow, L. J. Ptacek, D. Rand, A. B. Reddy, M. S. Robles, T. Roenneberg, M. Rosbash, M. D. Ruben, S. S. Rund, A. Sancar, P. Sassone-Corsi, A. Sehgal, S. Sherrill-Mix, D. J. Skene, K.-F. Storch, J. S. Takahashi, H. R. Ueda, H. Wang, C. Weitz, P. O. Westermark, H. Wijnen, Y. Xu, G. Wu, S.-H. Yoo, M. Young, E. E. Zhang, T. Zielinski, J. B. Hogenesch, Guidelines 
[29] B. Schwanhäusser, D. Busse, N. Li, G. Dittmar, J. Schuchhardt, J. Wolf, W. Chen, M. Selbach, Global quantification of mammalian gene expression control, Nature 473 (2011) 337-342. 
[30] C. Vogel, E. M. Marcotte, Insights into the regulation of protein abundance from proteomic and transcriptomic analyses, Nat Rev Genet 13 (2012) 227-232. doi:10.1038/nrg3185.

[31] G. M. Silva, C. Vogel, Quantifying gene expression: the importance of being subtle, Molecular Systems Biology 12 (2016) 885. doi:10.15252/msb.20167325.

[32] A. G. Hinnebusch, I. P. Ivanov, N. Sonenberg, Translational control by 5'-untranslated regions of eukaryotic mrnas, Science 352 (2016) 1413-1416. doi:10.1126/science.aad9868.

[33] C. C. Thoreen, L. Chantranupong, H. R. Keys, T. Wang, N. S. Gray, D. M. Sabatini, A unifying model for mTORC1-mediated regulation of mRNA translation, Nature 485 (2012) 109-113. doi:10.1038/nature11083.

[34] A. C. Hsieh, Y. Liu, M. P. Edlind, N. T. Ingolia, M. R. Janes, A. Sher, E. Y. Shi, C. R. Stumpf, C. Christensen, M. J. Bonham, S. Wang, P. Ren, M. Martin, K. Jessen, M. E. Feldman, J. S. Weissman, K. M. Shokat, C. Rommel, D. Ruggero, The translational landscape of mtor signalling steers cancer initiation and metastasis, Nature 485 (2012) 55-61. doi:10.1038/nature10912.

[35] R. Lacerda, J. Menezes, L. Romão, More than just scanning: the importance of capindependent mrna translation initiation for cellular stress response and cancer, Cellular and Molecular Life Sciences 74 (2017) 1659-1680.

[36] M. Kozak, An analysis of vertebrate mRNA sequences: intimations of translational control., Journal of Cell Biology 115 (1991) 887-903. doi:10.1083/jcb.115.4.887.

[37] S. Luck, K. Thurley, P. F. Thaben, P. O. Westermark, Rhythmic Degradation Explains and Unifies Circadian Transcriptome and Proteome Data, Cell Reports 9 (2014) 741-751. doi:10.1016/j.celrep.2014.09.021.

[38] M. J. Deery, E. S. Maywood, J. E. Chesham, M. Sládek, N. A. Karp, E. W. Green, P. D. Charles, A. B. Reddy, C. P. Kyriacou, K. S. Lilley, M. H. Hastings, Proteomic analysis reveals the role of synaptic vesicle cycling in sustaining the suprachiasmatic circadian clock, Current Biology 19 (2009) 2031-2036. doi:10.1016/j.cub.2009.10.024.

[39] C.-K. Chiang, N. Mehta, A. Patel, P. Zhang, Z. Ning, J. Mayne, W. Y. L. Sun, H.Y. M. Cheng, D. Figeys, The proteomic landscape of the suprachiasmatic nucleus clock 
reveals large-scale coordination of key biological processes, PLoS Genet. 10 (2014) e1004695. doi:10.1371/journal.pgen.1004695.

[40] S. Kojima, E. L. Sher-Chen, C. B. Green, Circadian control of mRNA polyadenylation dynamics regulates rhythmic protein expression, Genes Dev. 26 (2012) 2724-2736.

[41] J. R. Warner, P. M. Knopf, A. Rich, A multiple ribosomal structure in protein synthesis, Proceedings of the National Academy of Sciences of the United States of America 49 (1963) 122-129. 13998950[pmid], doi:10.1073/pnas.49.1.122.

[42] N. T. Ingolia, S. Ghaemmaghami, J. R. S. Newman, J. S. Weissman, Genome-Wide Analysis in

[48] V. Castelo-Szekely, A. B. Arpat, P. Janich, D. Gatfield, Translational contributions to tissue specificity in rhythmic and constitutive gene expression, Genome Biology 18 (2017) 116. doi:10.1186/s13059-017-1222-2. 
[49] R. Elfakess, H. Sinvani, O. Haimov, Y. Svitkin, N. Sonenberg, R. Dikstein, Unique transla-

[58] H. Reinke, C. Saini, F. Fleury-Olela, C. Dibner, I. J. Benjamin, U. Schibler, Differential display of dna-binding proteins reveals heat-shock factor 1 as a circadian transcription factor, Genes \& Development 22 (2008) 331-345. doi:10.1101/gad.453808. 
[59] C. Saini, J. Morf, M. Stratmann, P. Gos, U. Schibler, Simulated body temperature rhythms reveal the phase-shifting behavior and plasticity of mammalian circadian oscillators, Genes \& Development 26 (2012) 567-580. doi:10.1101/gad.183251.111.

[66] C. Vollmers, S. Gill, L. DiTacchio, S. R. Pulivarthy, H. D. Le, S. Panda, Time of feeding and the intrinsic circadian clock drive rhythms in hepatic gene expression, Proc Natl Acad Sci U S A 106 (2009) 21453-21458. doi:10.1073/pnas.0909591106. 
[67] F. Mange, V. Praz, E. Migliavacca, I. M. Willis, F. Schütz, N. Hernandez, the CycliX Consortium, Diurnal regulation of rna polymerase iii transcription is under the control of both the feeding-fasting response and the circadian clock, Genome Research 27 (2017) 973-984. doi:10.1101/gr.217521.116.

[68] B. J. Greenwell, A. J. Trott, J. R. Beytebiere, S. Pao, A. Bosley, E. Beach, P. Finegan,

[70] B. Fishman, R. J. Wurtman, H. N. Munro, Daily rhythms in hepatic polysome profiles and tyrosine transaminase activity: role of dietary protein, Proc. Natl. Acad. Sci. U.S.A. 64 (1969) $677-682$.

[71] F. Sinturel, A. Gerber, D. Mauvoisin, J. Wang, D. Gatfield, J. J. Stubblefield, C. B. Green,

[74] M. Hatori, C. Vollmers, A. Zarrinpar, L. DiTacchio, E. Bushong, S. Gill, M. Leblanc, A. Chaix, M. Joens, J. J. Fitzpatrick, M. Ellisman, S. Panda, Time-Restricted Feeding without Reducing Caloric Intake Prevents Metabolic Diseases in Mice Fed a High-Fat Diet, Cell Metabolism 15 (2012) 848-860. doi:10.1016/j.cmet.2012.04.019.

[75] D. M. Arble, J. Bass, A. D. Laposky, M. H. Vitaterna, F. W. Turek, Circadian Timing of Food Intake Contributes to Weight Gain, Obesity 17 (2009) 2100-2102. doi:10.1038/oby.2009.264. 
[76] R. Salgado-Delgado, M. Angeles-Castellanos, N. Saderi, R. M. Buijs, C. Escobar, Food intake during the normal activity phase prevents obesity and circadian desynchrony in a rat model of night work, Endocrinology 151 (2010) 1019-1029. doi:10.1210/en.2009-0864.

[77] D. A. Golombek, L. P. Casiraghi, P. V. Agostino, N. Paladino, J. M. Duhart, S. A. Plano, J. J. Chiesa, The times they're a-changing: Effects of circadian desynchronization on physiology and disease, Journal of Physiology-Paris 107 (2013) 310-322. doi:10.1016/j.jphysparis.2013.03.007.

[78] V. D. Longo, S. Panda, Fasting, Circadian Rhythms, and Time-Restricted Feeding in Healthy Lifespan, Cell Metab. 23 (2016) 1048-1059. doi:10.1016/j.cmet.2016.06.001.

[79] A. Chaix, T. Lin, H. D. Le, M. W. Chang, S. Panda, Time-Restricted Feeding Prevents Obesity and Metabolic Syndrome in Mice Lacking a Circadian Clock, Cell Metab. 29 (2019) 303-319.e4. doi:10.1016/j.cmet.2018.08.004.

[80] J. Lipton, E. Yuan, L. Boyle, D. Ebrahimi-Fakhari, E. Kwiatkowski, A. Nathan, T. Guttler, F. Davis, J. Asara, M. Sahin, The Circadian Protein BMAL1 Regulates Translation in Response to S6k1-Mediated Phosphorylation, Cell 161 (2015) 1138-1151. doi:10.1016/j.cell.2015.04.002.

[81] R. Wu, F. Dang, P. Li, P. Wang, Q. Xu, Z. Liu, Y. Li, Y. Wu, Y. Chen, Y. Liu, The circadian protein period 2 suppresses mtorc1 activity via recruiting tsc1 to mtorc1 complex, Cell Metabolism 29 (2019) 653 - 667.e6. doi:10.1016/j.cmet.2018.11.006.

[82] C. Ramanathan, N. D. Kathale, D. Liu, C. Lee, D. A. Freeman, J. B. Hogenesch, R. Cao, A. C. Liu, mtor signaling regulates central and peripheral circadian clock function, PLOS Genetics 14 (2018) 1-21. doi:10.1371/journal.pgen.1007369.

[83] J. O. Lipton, L. M. Boyle, E. D. Yuan, K. J. Hochstrasser, F. F. Chifamba, A. Nathan, P. T. Tsai, F. Davis, M. Sahin, Aberrant proteostasis of bmal1\&\#xa0;underlies circadian abnormalities in a paradigmatic mtor-opathy, Cell Reports 20 (2017) 868-880. doi:10.1016/j.celrep.2017.07.008.

[84] P. Crosby, R. Hamnett, M. Putker, N. P. Hoyle, M. Reed, C. J. Karam, E. S. Maywood, A. Stangherlin, J. E. Chesham, E. A. Hayter, L. Rosenbrier-Ribeiro, P. Newham, H. Clevers, D. A. Bechtold, J. S. O'Neill, Insulin/igf-1 drives period synthesis to entrain circadian rhythms with feeding time, Cell 177 (2019) 896-909.e20. doi:10.1016/j.cell.2019.02.017. 
[85] R. Cao, B. Lee, H. yeon Cho, S. Saklayen, K. Obrietan, Photic regulation of the mtor signaling pathway in the suprachiasmatic circadian clock, Molecular and Cellular Neuroscience 38 (2008) 312 - 324. ://doi.org/10.1016/j.mcn.2008.03.005.

[86] R. Cao, A. Li, H.-y. Cho, B. Lee, K. Obrietan, Mammalian target of rapamycin signaling

[93] A. C. Diernfellner, T. Schafmeier, M. W. Merrow, M. Brunner, Molecular mechanism of temperature sensing by the circadian clock of neurospora crassa, Genes\& Development 19 (2005) 1968-1973. doi:10.1101/gad.345905. 
[94] S. Schleich, K. Strassburger, P. C. Janiesch, T. Koledachkina, K. K. Miller, K. Haneke, Y.-S.

[102] G. Hanson, J. Coller, Codon optimality, bias and usage in translation and mRNA decay, Nature Reviews. Molecular Cell Biology 19 (2018) 20-30. doi:10.1038/nrm.2017.91. 